\title{
Variable offspring size as an adaptation to environmental heterogeneity in a clonal plant species: integrating theory and data
}

4 5
1: Centre D’Ecologie Fontionnelle et Evolutive, CNRS, Montpellier, France

2: Department of Environmental Biology, University of Guelph, Canada

3: Department of Mathematics and Statistics, University of Guelph, Canada

\#: these authors contributed equally to the work

*: author to whom correspondence should be addressed

email: manand@uoguelph.ca

fax: $519837-0442$

Running head: Bet-hedging strategies in clonal plants

Submission type: Standard paper

\author{
Anne Charpentier ${ }^{1 \#}$, Madhur Anand ${ }^{2 \#^{*}}$, Chris T. Bauch ${ }^{3 \#}$
}




\section{Summary}

1. The production of variably sized offspring has been hypothesized to be adaptive to temporal variability in environmental conditions.

2. This is difficult to verify empirically, and theoretical models are typically generic and not parameterized with data from real populations; studies integrating theoretical and empirical approaches to this problem are rare.

3. Here, we present field and experimental data on the growth of Scirpus maritimus, a clonal aquatic macrophyte that grows through vegetative extensions involving tubers.

4. The experiments show that offspring fitness (biomass productivity) is dependent on environmental conditions (water depth).

5. Both field and experimental data indicate that variation in offspring (tuber) sizes of individual ramets can be approximated by a lognormal distribution.

6. We use these data to develop a difference equation model of S. maritimus growth in order to test whether producing variably sized offspring is adaptive. The model compares fitness under the lognormal strategy to several hypothetical strategies with qualitatively different variance in offspring size.

7. The model results suggest that lognormal variation in S. maritimus tuber size may be adaptive to the temporal variation in water levels that characterize its natural Mediterranean environment.

8. We illustrate how the underlying principles that lend adaptive value to offspring size variation may apply to other species experiencing similar environmental conditions. investigating the adaptive value of life history traits in clonal aquatic plans. 
2 Keywords: Scirpus maritimus, mathematical model, size-number tradeoff, environmental

3 variability, theoretical model, lognormal distribution, bet-hedging

\section{Introduction}

8 One of the central issues that have informed life history theory is the tradeoff between size 9 and number of offspring (Stearns 1992, Moles \& Westoby 2006). For a given level of resource investment, a parent may choose to produce a few large-sized offspring (with higher

11 individual probabilities of survival to reproductive maturity) or many small-sized offspring 12 (with lower probabilities of survival). A standard mathematical model predicts the evolution 13 of a single optimal offspring size that maximizes maternal fitness, in situations where 14 offspring fitness increases with size according to a certain functional form (Smith \& Fretwell 1974). Subsequent work has extended this model and related it to ecologically important 16 phenomena including competition, dispersal, predation, and host-parasite relationships (Parker \& Begon 1986, Venable 1992, Fox et al. 1997, Marshall et al. 2006).

One such extension investigates whether the single-sized offspring strategy is still optimal when the environment varies unpredictably in time or in space. In this case, an alternative to a

21 single-size strategy is for an individual to produce variably sized offspring. This may provide

22 a buffer against environmental variability and constitutes a form of bet hedging (Philippi \& 23 Seger 1989, Marshall et al 2008, Olofsson et al 2009). Since the 1970s various authors have 24 described offspring size variability in a large range of taxa among plants, insects, fishes, birds, 25 vertebrate and suggested its possible adaptive value in variable habitats (for references, see 
1 McGinley et al. 1987, Geritz 1995, Geritz et al. 1999, Westoby et al. 1996, Moles \& Westoby

2 2006). However, variable offspring size within a species can arise from a number of genetic

3 and environmental sources and can be partitioned in numerous ways: among populations,

4 among individuals in the same population, within individuals, within a year or a clutch, or

5 between years (Hangelbroek \& Santamaria 2004). As a consequence it is often difficult to

6 determine if the observed offspring size variation simply reflects developmental or resource

7 constraints or whether it might also have adaptive value (Sakai 1995, Wolfe 1995, Simons \&

8 Johnston 1997, Vinuela 1997, Vaughton \& Ramsey 1998, Forbes 1999, Simons \& Johnston

9 2000, Einum \& Fleming 2004). A second obstacle to establishing the adaptive value of

10 offspring size variation is that the stipulation of specific functional forms relating fitness to

11 offspring size and number is often difficult to establish (Einum \& Fleming 2000, Torres-Vila

12 \& Rodriguez-Molina 2002, Moles et al 2003, Gómez 2004, Moles \& Westoby 2006). For

13 example, it is generally admitted that the survival of seedlings increases with seed size,

14 however large seeds can also have higher risks of predation or reduced dispersal, resulting in a

15 co-evolution of traits (Venable \& Brown 1988, Moles et al 2003). The relationship between

16 offspring size and fitness may also vary between different stages of development or depend on

17 parental behavior (Hendry et al. 2001). Hence, although there have been several empirical

18 studies indicating that variation in offspring size can indeed be an adaptive response to

19 unpredictable environmental variation (Fox et al. 1997, Kudo 2001), empirical support for the

20 hypothesis remains rare and largely hypothetical. Such obstacles also prevent the forging of

21 more intimate links between theoretical and empirical approaches to the problem.

23 There have been a number of theoretical modeling studies of strategies for offspring size

24 distribution and/or offspring numbers in the presence of temporally or spatially varying 25 environments (Kaplan and Cooper 1984, Schultz 1991, Geritz 1995, Sasaki and Ellner 1995, 
1 Geritz et al 1999, Kisdi 2007, Simons 2007, Olofsson et al 2009). The process generally

2 followed by mathematical models is to compare the fitness of a single-size strategy to a

3 strategy of producing variably sized offspring under different conditions of habitat variability

4 in space and/or time. Most of these theoretical approaches conclude that, under certain

5 assumptions, the optimal solution to the tradeoff between size and number of offspring in a

6 spatially and/or temporally variable environment is the production of variably sized offspring

7 (Kaplan and Cooper 1984, Schultz 1991, Geritz 1995, Geritz et al 1999). However, one

8 modeling study concluded that variability in offspring size does not necessarily provide a

9 fitness advantage over single-size strategies in a temporally varying environment that can be

10 either "good" or "bad" (McGinley et al 1987). These studies have generally not

11 parameterized their models with empirical data, aiming for generality instead.

13 Another possible adaptive response to temporally varying environmental conditions is 14 dormancy, and several studies have used empirical data and/or models parameterized with 15 empirical data to investigate this issue (Simons \& Johnston 2006; Venable 2007). For 16 example, two studies that parameterize a simulation model with empirical data suggest that 17 seed banking may be an adaptive response to temporally variability in environmental 18 conditions (Kalisz and McPeek 1993, Evans et al 2007). Another study parameterizes a 19 simulation model of marine copepods using field data to determine evolutionarily stable 20 diapause strategies in a temporally varying environment (Hairston \& Munns 1984). In 21 contrast, a modeling study by Einum \& Fleming (2004) based on data from Atlantic salmon 22 found that within-clutch variability more likely reflects difficulties in allocating resources 23 equally among offspring rather than evolutionary adaptation per se. 
1 Studies that parameterize theoretical models with empirical data in order to investigate the

2 adaptive value of producing variably sized offspring in temporally varying environments

3 remain uncommon. Here, we describe experiments carried out to investigate offspring size

4 variation in a clonal plant species and use the experimental data to develop a species-specific

5 mathematical model of growth dynamics. In particular, we (1) experimentally determine the

6 relationships between offspring size and fitness in the clonal plant Scirpus maritimus under

7 different environmental conditions, and (2) use these experimental data, together with

8 longitudinal historical data on the temporally varying environment of $S$. maritimus, to develop

9 a difference equation model of $S$. maritimus growth dynamics. The model allows us to

10 compare the fitness under the offspring size distribution observed in natural populations to the

11 fitness under several hypothetical alternative offspring size distributions, and under different

12 conditions of temporal environmental variability. The objective is to determine whether the

13 observed variation in S. maritimus offspring sizes is adaptive to a temporally varying

14 environment. This integrated approach allows us to address issues that could not be addressed

15 with empirical or theoretical approaches in isolation from one another. In addition to its

16 relevance to the theory of parental investment, this question is also important to ecology

17 because its answer could shed light on the potential geographic extent of the species (e.g., its

18 ability to colonize areas with highly variable rainfall/water levels) or how it might respond to

19 environmental change it is present habitat. Finally, by conducting further simulations, we also

20 investigate the impact of representing environmental variability through a discrete number of

21 possible states, rather than a continuous distribution of possible states. In the following

22 section we describe materials and methods (for field data, experimental, and modelling

23 components of the study), results (for field data, experimental, and modelling components),

24 and we conclude with a discussion. 


\section{Materials and methods}

\section{STUDY SPECIES}

4

5 We investigate the adaptive value of variable offspring size in the emergent macrophyte

6 Scirpus maritimus. The species grows clonally by producing underground sympodial 7 rhizomes. The initial above-ground shoot of a seedling produces from one to three rhizomes at 8 its base which grow horizontally and turn upwards to form erect above-ground shoots. Each 9 new above-ground shoot then produces from one to three new rhizomes. Branched systems composed of several tens of sympodial rhizomes can be produced from an initial shoot during

11 a single growing season (Clevering 1995, Charpentier et al. 1998). During the growing season each shoot forms a tuber at its base. Among the tubers produced in a single growing season, tuber biomass increases from the early- to the later-produced tubers along rhizome 14 systems (Lieffers \& Shay 1982, Clevering 1995, Charpentier \& Stuefer 1999). Clonal growth 15 stops at the end of summer. Aboveground shoots dry out and die in the autumn, while tubers 16 persist in the soil. In spring, new shoots are produced from axillary buds situated on overwintering tubers and start to produce from one to three rhizomes.

19 Several studies have described in detail the variation in tuber size in this species (Lieffers \& 20 Shay 1982, Clevering 1995, Charpentier et al. 1998, Charpentier \& Stuefer 1999). The 21 variation arises within individuals during a single season: tuber size increases gradually from the first to the last produced tubers. This pattern is observed in the field as well as in single

23 plants grown in pots under stable conditions, suggesting that this variation can be attributed to

24 a developmentally programmed syndrome rather than environmental stress or changes in 25 environmental conditions during the season. 
2 The ecology of this species allows us to expect a strong relationship between size and fitness

3 of offspring. In emergent macrophytes, because of the low efficiency of underwater

4 photosynthesis, it is generally assumed that the maximum water depth they can overcome in

5 spring depends on the amount of reserve stored in overwintering organs (e.g. tubers,

6 rhizomes) (Spencer 1982, Grace 1989, Clevering et al. 1995). We can thus suppose that in $S$.

7 maritimus, the ability of shoots to emerge above the water surface in spring is crucial for

8 survival and depends on tuber size, i.e. the amount of stored reserves. (Nutrient availability,

9 particularly that of oxygen, is also reduced at greater depths, and therefore larger tubers with

10 more reserves are more likely to be able to reach shallow depths where more nutrients are

11 available.) Therefore, (1) we can use offspring tuber biomass as an analog for vegetative

12 offspring size in S. maritimus, (2) we can use water depth as an analog for environmental

13 quality, and (3) we can use total tuber biomass produced by an initially present tuber during a

14 growth season as an analog for fitness of the initial tuber. The water regime of wetlands that

15 support $S$. maritimus worldwide are variously described as irregularly exposed, temporary,

16 irregular, seasonally and permanently flooded, all characterized by a strong variability of

17 water levels (Kantrud 1996). Therefore, we can also suppose that the variability and

18 unpredictability of water regime represents a strong environmental pressure for the evolution

19 and ecology of this species.

FIELD DATA

24 To examine the natural variability in offspring tuber sizes in S. maritimus, 550 tubers were 25 collected from a population in the wildlife reserve of the Tour du Valat, Rhone delta, 
1 Southern France in autumn 1998. Tubers were stored under moist dark conditions at $5^{\circ} \mathrm{C}$. In

2 spring 1999, the fresh weight of all the tubers was measured. The size distribution was fit to

3 several functions to determine the best goodness of fit using the statistical software program

$4 \quad \mathrm{JMP}$ (version 5.1 for Macintosh).

6 The population was located in a temporary marsh in a region that experiences strong between7 year variation in water level due to the unpredictable rainfall patterns characteristic of a 8 Mediterranean climate. Rainfall data for 1932-2003 were obtained from a meteorological 9 station a few kilometers from the marsh. The water depth in the marsh was monitored from 1995 to 2003. The relationship between the total rainfall from September to March and the

11 water level at the end of March (start of the growing season) was determined for 1995-2003 12 (Supporting Information, Fig. A1). Using this relationship, the yearly water level was then 13 derived for the period 1932-2003 based on the historical rainfall data for 1932-2003. The 14 frequency distribution of water levels was fit by several distributions using the statistical 15 software program JMP (version 5.1 for Macintosh) and MATLAB (version for Macintosh).

EXPERIMENT

An experiment was performed at Utrecht University in 1999 to test the hypothesis that biomass productivity of $S$. maritimus is dependent on environmental quality and the size of the initial tuber. Tubers collected from the field in autumn 1998 were stored under moist dark conditions at $5^{\circ} \mathrm{C}$, and in spring 1999 , the fresh weight of all the tubers was measured. Three series of 30 tubers from the tubers collected from the field (see previous section) with the

24 following fresh weight $(\mathrm{g})$ were selected for the experiment: $0.5,0.6,0.9,1.0,1.1,1.5,1.6$, 25 $1.9,2.0,2.1,2.5,2.6,2.9,3.0,3.1,3.5,3.6,3.9,4.0,4.1,4.9,5.0,5.1,5.9,6.0,6.1,7.0,7.1$, 
$18.0,10.0$. The dry weight of the remaining 450 tubers was measured to establish the fresh-dry

2 weight relationship (freshweight $=b^{*}$ dryweight $+a$, where $b=0.01$ and $a=3.25 p<0.05$ ). On

3 May 17, 1999 the three series of tubers were randomly allocated to one permanent water level

4 treatment: shallow $(15 \mathrm{~cm})$, medium $(45 \mathrm{~cm})$ or deep $(85 \mathrm{~cm})$. Single tubers were planted at 2

$5 \mathrm{~cm}$ depth in pots of diameter $21 \mathrm{~cm}$, filled with a 1:2 compost-sand mixture. The pots were

6 put outside into containers under the three permanent water levels above the soil surface. In

7 Amsterdam from May-September 1999, key weather indices determining the growth

8 environment of the tubers were: mean air temperature 16.5 degrees Celsius, mean humidity

$976.2 \%$, total precipitation $41.1 \mathrm{~cm}$, and mean wind speed $16.2 \mathrm{~km} / \mathrm{h}$. In September 1999, the

10 dry weight of every tuber produced by each planted tuber was recorded. For each planted

11 tuber, the mean and standard deviation of its offspring tuber weights were calculated.

12 Moreover, for those planted tubers with a sufficiently large number of offspring, the offspring

13 size distribution was fit to several candidate functions using the statistical software package

14 JMP (version 5.1 for Macintosh) and goodness-of-fit for the functions was assessed, in order

15 to characterize the offspring size distribution of individual tubers. The size distribution of the

16 pooled offspring tubers was also fitted in JMP for each water level, thus characterizing

17 population-level distribution of offspring tuber sizes.

19 We treat the total biomass produced by an initial tuber as a proxy for the tuber's fitness. To 20 quantify the relationship between fitness and offspring size, we fit the data from the 21 experiments to a commonly used fitness function (e.g. Smith \& Fretwell 1974): offspring 22 fitness $F_{\mathrm{w}}$ in a given water level $w$ is related to the size $s$ of the offspring via

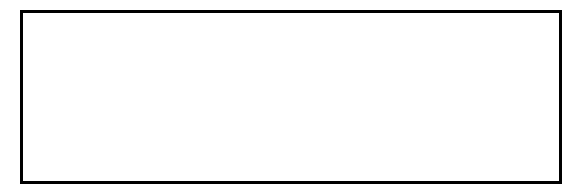


1 where $m_{\mathrm{w}}$ is the minimum viable offspring size in water level $w$ and $\alpha_{\mathrm{w}}$ is a constant

2 determining how quickly the fitness curve approaches its asymptotic value as $s$ increases. In

3 the special case where the environmental quality is constant over time, the optimal offspring

4 size $s^{*}$ can be obtained from equation 1 (Smith \& Fretwell 1974):

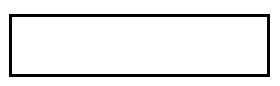

6 We computed the best fit of the experimental results to equation 1 for each of the three environmental conditions using the nonlinear-fitting algorithm in JMP, and setting maximum observed biomass produced as the maximum fitness level. Hence, we obtained three sets of $m_{\mathrm{w}}$ and $\alpha_{\mathrm{w}}$ values for each of the three experimental water levels.

\section{MATHEMATICAL MODEL}

We used the empirical data to parameterize a mathematical (difference equation) model of

14 biomass production in a single genet of $S$. maritimus over multiple seasons. The goal was to 15 compare fitness under the observed offspring size distribution to fitness under two hypothetical alternatives under several environmental scenarios, and thus to test the hypothesis that the observed variation in S. maritimus offspring tuber size is adaptive to a temporally varying environment. We chose the route of "simulation as experiment" because 19 of the difficulty of testing this hypothesis empirically (Peck 2004).

21 We derived a discrete-time difference equation model of the temporal dynamics of total biomass production under the three strategies for some given temporal variation in water level. The total biomass produced in the current year depends in a simple way upon how many tubers were present in the genet at the beginning of the growth season (produced the 25 year previous), their size distribution, and how much biomass a tuber of a given size can 
1 produce as dependent on this year's average water level. Mathematically, the total biomass $b_{\mathrm{t}}$

2 produced in year $t$ is related to the biomass $b_{\mathrm{t}-1}$ produced the year previous and the water levels $3 \quad w(t)$ and $w(t-1)$ in years $t$ and $t-1$, by the equation

5 where $\square$ is the mean tuber size produced in year $t-1, \phi_{\mathrm{w}(\mathrm{t})}(s)$ is the offspring size distribution,

6 i.e., the probability of producing an offspring of size $s$ when the average water level in year $t$

7 is $w(t)$, and $F_{w(t)}(s)$ is the fitness function, equation 1 (see Supporting Information for

8 derivation). S. maritimus ramets may die through excessive water depth. This source of

9 mortality is represented in equation 1 since no biomass is produced in year $t$ if all tubers

10 produced in year $t-1$ are smaller than the minimal offspring size $m_{\mathrm{w}(\mathrm{t})}$ for year $t$. This happens

11 if there is a string of years with sufficiently high water levels. To account for other causes of

12 mortality (such as herbivory, disease), we assumed a $1 \%$ probability of genet death each year

13 from other causes.

14

15 denote the ratio of biomass produced in year $t$ to biomass produced in year $t$ -

1. This quantity is analogous to fecundity, in the sense that it must remain above one for the genet to experience net growth over multiple seasons. From equation $3, R_{\mathrm{t}}$ is given by

Then, we define fitness as the geometric mean of the values of $R_{\mathrm{t}}$ for $t=1 \ldots T$, where $T$ is the lifespan of the genet in years. Thus

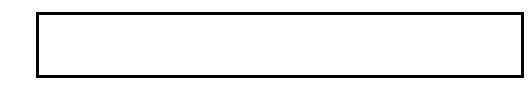

The geometric mean is a good measure of fitness in situations where environmental conditions vary from year to year (McGinley et al 1987), because the geometric mean would 
1 indicate zero fitness for a genet that died before time $T$, whereas the arithmetic mean would

2 indicate positive fitness in the same situation.

4 We carried out four sets of simulation experiments to test the hypothesis that offspring tuber

5 size variation has adaptive value in $S$. maritimus, and to investigate the relationship between

6 our predictions and previous models that predict a single optimal offspring size (McGinley et

7 al 1997; Smith \& Fretwell 1974). We compared geometric mean fitness and lifespan of 8 genets under the three offspring size distributions. For each given set of parameter values, a 9 large number of realizations were carried out (8,000 for experiments 1,2 and 4 and 5,000 for experiment 3), starting from the same initial conditions. The resulting geometric fitness over 11350 years was averaged over the realizations. The simulation experiments are described in 12 greater detail in Results.

15 Results

\section{FIELD DATA}

The distribution of tuber sizes in the field appears to be qualitatively consistent with the lognormal distribution (Fig. 1A). A quantity $X$ is lognormally distributed if $\log (X)$ is normally

21 distributed (Limpert et al 2001). The lognormal distribution is given by , where $\mu$ is the mean of $\ln (X)$ and $\sigma$ is the variance of $\ln (X)$.

23 Though not found to be statistically significant, the best-fit parameters (Kolmogorov test:

$24 D=0.06, p=0.01$ ) for the tuber sizes from the field data were when $\mu=-0.636$ and $\sigma=0.821$

25 (corresponding to a mean tuber size of $0.529 \mathrm{~g}$ and a variance of 2.27). The frequency 
1 distribution of the water level $w$ from to 1932 to 2003 (Fig. 2A) is well-described by a

2 function of the form

4 The best-fit $\left(r^{2}=0.937, p<0.001\right)$ parameters were found to be $a=2.323, b=0.080$. This function 5 provided a better fit than better-known distributions such as Gamma and lognormal.

\section{EXPERIMENT}

Water level had a significant effect on tuber survival. In the shallow treatment, all planted tubers survived and produced new tubers, while in medium and deep conditions all planted 11 tubers smaller than $0.33 \mathrm{~g}$ and $0.79 \mathrm{~g}$ respectively produced a shoot that never reached the 12 water surface, and died during the summer (Fig. 2B). The water level also affected the total biomass of newly produced tubers. Productivity was highest in shallow water and decreased

14 successively under medium and deep water levels (Fig. 2B).

After fitting equation 1 to the experimental data, the following parameter values for shallow, medium and deep respectively were obtained: $m_{15}=0.05 \mathrm{~g}, m_{45}=0.31 \mathrm{~g}, m_{85}=0.77 \mathrm{~g}, \alpha_{15}=0.22$, $\alpha_{45}=0.17, \alpha_{85}=0.07(p<0.01)$. A least-squares regression between the three values of $m_{\mathrm{w}}$ and water level $w$ fits the data very well $\left(\mathrm{r}^{2}=0.99, \mathrm{p}<0.01\right)$

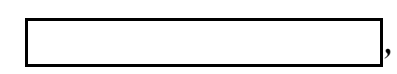

21 and the fit between the three values of $\alpha_{\mathrm{w}}$ and $w$ is also significant $\left(\mathrm{r}^{2}=0.98, \mathrm{p}<0.05\right)$,

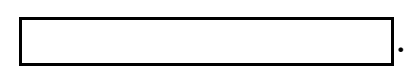

24 Using the parameterized fitness functions, we can estimate the optimal offspring tuber size $s^{*}$ from equation 2 for a clonal plant species in a hypothetical environment with unchanging 
1 water level: $s^{*}=0.08 \mathrm{~g}$ for shallow, $s^{*}=0.85 \mathrm{~g}$ for medium, and $s^{*}=1.97 \mathrm{~g}$ for deep water

2 levels. For an unchanging water level of $25.5 \mathrm{~cm}$, which is the average water level in the field

3 as computed from equation 7 , the optimal tuber size would be $s^{*}=0.332 \mathrm{~g}$. In absolute terms

4 this is somewhat below the observed mean $(0.533 \mathrm{~g})$ and median $(0.505 \mathrm{~g})$ tuber size in the

5 field, although it is not clear what relationship should be expected between offspring tuber

6 sizes observed in field populations experiencing temporally-varying environmental quality and tuber sizes predicted from a model that assumes a constant environmental quality.

9 The offspring sizes of planted tubers were highly variable in all water level treatments (Supporting Information, Table A1). For each of the water treatment levels, we selected the 11 three planted tubers that produced the largest number of offspring tubers (as long as they 12 produced at least ten), and tested goodness-of-fit for lognormal, normal, and gamma 13 distributions fitted to the data. The lognormal distribution fit the size distribution of tubers 14 produced best for almost all individual ramets tested (Table 1). For the purposes of 15 parameterizing the simulation model, we also pooled individual data to determine estimates of 16 the parameters of the lognormal distribution. In the shallow treatment $(N=398)$ appeared to follow a lognormal distribution, but it was not statistically significant $(\mu=-0.788, \sigma=0.837$, Kolmogorov $D=0.05 ; p<0.01$ ) (Fig. 1B). The distribution for the medium $(N=210)$ and deep 19 treatments $(N=94)$ did statistically fit a lognormal distribution $(D=0.05 ; p=0.15$ and $D=0.07$; $p=0.15$, respectively) (Fig. $1 \mathrm{C}, \mathrm{D})$. Note that the variance $\sigma$ in all three water levels is similar 21 to that of the field population.

23 The mean size of the produced tubers was also significantly affected by water level. The 24 produced tubers were significantly larger in shallow $(\square=0.62 \mathrm{~g}$, S.E. $=0.02)$ than in medium $(\square=0.46$ g, S.E. $=0.03$ ) or deep water $(\square=0.30$ g, S.E.=0.03) (one way ANOVA on log- 
1 transformed data: $F=28.8, D F=2, p<0.001)$. The three data points relating mean tuber size 2 produced $(\square)$ to water level $(w)$ are well-fit by a linear regression $\left(\mathrm{r}^{2}=0.99, \mathrm{p}<0.01\right)$ :

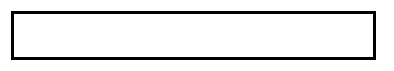

\section{MATHEMATICAL MODEL}

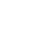

We compared biomass production under the observed lognormal strategy to two hypothetical alternative strategies: the single-size distribution, in which all offspring are the same size, and the uniform distribution, in which the probability of producing tubers of a given size $x$ is a constant, nonzero value over a given interval of sizes $\left[x_{\text {lower }}, x_{\text {upper }}\right]$, and is zero outside the interval (Supporting Information, Fig. A2 and equation A1). The single-size distribution corresponds to the "optimal offspring size" theory and thus should be optimal in an environment without temporal variation. The uniform distribution represents an intermediate strategy between the single-size and lognormal strategies. These alternative strategies act as theoretical controls for the lognormal strategy. The three distributions together represent a broad possible range of resource allocation strategies: (1) low variance, where all offspring have the same size (single-size strategy), (2) intermediate variance, where offspring of various sizes can be produced but only over a restricted interval of sizes (uniform strategy), and (3) high variance, where there is a nonzero probability of offspring of any size being produced (lognormal strategy).

Simulation experiment \#1: fitness at empirical parameter values. In simulation experiment \#1, we compared the fitness of the three strategies when the model parameters are set to match the empirically observed values, and where the water level varies from year to year according to patterns observed in the field. The water level $w(t)$ in a given year $t$ was a random variate sampled from equation 7 at best-fit parameter values, normalized to yield a probability density 
1 function. Then, $\alpha_{\mathrm{w}}, m_{\mathrm{w}}$ and $\square$ for the sampled water level $w(t)$ were determined from

2 equations $8-10$. For $w<12.01 \mathrm{~cm}$ in equation 8 , we set $m_{\mathrm{w}}=0$ since otherwise $m_{\mathrm{w}}$ is negative;

3 likewise for $w>114.91 \mathrm{~cm}$ in equation 9 we set $\alpha_{\mathrm{w}}=0$ since otherwise $\alpha_{\mathrm{w}}$ is negative. Thence,

4 fitness $F_{w}(s)$ was determined from equation 1 and $\phi_{w}(s)$ from equation A1 (Supporting

5 Information). Finally, total biomass $b_{\mathrm{t}}$ produced during the season was determined from 6 equation 3.

8 For a given water level $w$, the three strategies produce the same mean tuber size $\square$ given by 9 equation 10. Thus, the strategies differ only in the way that tuber offspring size is distributed 10 about the mean, and any difference in strategy fitness arises from the variance $\sigma$ of the 11 offspring size distribution. For the single-size strategy, $\sigma=0$ by definition. For the lognormal 12 strategy, $\sigma$ did not vary widely across the three experimental treatments and the field data, 13 hence we used $\sigma=0.821$ in the simulations, which is the field value and also very close to the 14 average of the three values of $\sigma(0.817)$ from the three experimental treatments. For the 15 uniform strategy, $\sigma$ is determined by $s_{\text {half }}$; we used $s_{\text {half }}=0.1$ and considered only $\square>s_{\text {half. }}$ The 16 value $s_{\text {half }}=0.1$ was chosen because it produces a distribution broad enough to be meaningfully 17 different from the single-size distribution, yet narrow enough to allow a large range of mean 18 tuber sizes to be tested (everything up to $\square=0.1$ ). All baseline parameter values for this and 19 subsequent simulation experiments are summarized in Table 2.

21 At the empirical parameter values, the lognormal strategy had the highest average fitness 22 across all realizations (mean 663.6, SD 622.9), followed by the uniform strategy (mean 327.9, 23 SD 362.7) and the single-size strategy (mean 229.6, SD 240.6). The lognormal strategy had 24 the highest fitness in $56 \%$ of realizations, followed by the uniform strategy $(24 \%)$ and the 25 single-size strategy (18\%). The distribution of mean geometric fitness $K$ over all realizations 
1 appears to be approximately exponential (Fig. 3A), which is why the mean approximately

2 equals the standard deviation. Accordingly, lifespan tends to be distributed exponentially as

3 well (Fig. 3B). Exponentiality arises from the assumption of a constant probability of death

4 due to causes other than excessive water depth. Under the lognormal strategy, genets also had

5 the longest mean lifespan (mean 89.6 years, SD 84.0), followed by the uniform (mean 36.6,

$6 \quad$ SD 37.3 ) and single-size strategies (mean 23.6, SD 24.4).

Simulation experiment \#2: fitness landscape. If the observed lognormal variation in offspring size is indeed adaptive to temporally-varying environmental conditions, then the fitness of the lognormal strategy at the empirical parameter values should be higher than the fitness at other

11 hypothetical parameter values to which the organism is free to evolve toward. Therefore, in simulation experiment $\# 2$, we examined fitness of the three strategies across a range of parameter values in order to determine whether the empirical values fall near the global maximum of the fitness landscape. The lower bound for $\sigma$ was zero. The upper bound for $\sigma$ was chosen as the value above which discrete effects invalidate the use of the model (see Supporting Information). Accordingly, $\sigma$ was varied in the range $[0,1.5]$ in increments of 0.1 to explore the fitness landscape. We also varied the slope of the linear relationship between mean tuber size produced and water level, equation 10. This corresponds to a tradeoff 19 between producing many small tubers or a few large tubers. The water level at which no tubers are produced (the x-intercept of equation 10) was fixed at $137.7 \mathrm{~cm}$. We assumed the

21 inability to produce tubers beyond this depth to be an absolute constraint on the organism.

22 Hence, we varied the slope of equation 10 by changing the y-intercept $\left(y_{\text {int }}\right)$. High $y_{\text {int }}$ means 23 that fewer, but larger, tubers are produced. For all three strategies $y_{\text {int }}$ was varied in the range $24[0,2]$ in increments of 0.05 . Beyond $y_{\text {int }}=2$, fitness approaches zero monotonically and 25 discrete effects may become important (see Supporting Information). As in simulation 26 experiment \#1, the water level was sampled from equation 7 and we used equations $3,8-10$ to 
1 model biomass dynamics. Together, the parameters $\sigma$ and $y_{\text {int }}$ control the most important

2 degrees of freedom in an organism's offspring size strategy: $\sigma$ controls the amount of

3 variability in offspring size and $y_{\text {int }}$ controls whether few large tubers or many small tubers are

4 produced. Therefore, a fitness landscape with $\sigma$ and $y_{\text {int }}$ on two axes should indicate whether selection is expected to occur with respect to those parameters.

7 Fig. 4A, B shows the fitness landscape of the lognormal distribution for range of $y_{\text {int }}$ and $\sigma$ 8 values described above. The fitness landscape has a single peak in the lower-right quadrant of 9 the parameter plane (dark red region): the maximum geometric mean fitness of 723.5 occurs at $y_{\text {int }}=0.5, \sigma=0.8$. The empirical parameter values $\left(y_{\text {int }}=0.671, \sigma=0.821\right)$ fall near this peak.

11 (This fitness at the peak is also higher than those obtained for the single-size and uniform 12 strategies.) The proximity of the empirical parameter values to the hypothetical values where 13 fitness is maximized is consistent with an adaptive response to temporal variation in water 14 level. The fitness changes more dramatically with changes in $y_{\text {int }}$ than with changes in $\sigma$, this 15 suggests that selection upon the size-number strategy is stronger than selection on the degree of variability in offspring size, although it is difficult to know how absolute changes in fitness for changing $\sigma$ maps to absolute strength of selection on variance in natural populations.

Simulation experiment \#3: fitness under a discrete number of possible environmental states.

Several studies have modeled temporal variability by assuming that environmental quality is sampled from a discrete number of possible environmental states (McGinley et al. 1987, Kalisz \& McPeek 1993). For instance, McGinley et al model fitness using equation 1 but only allow for two possible environmental states - "good" and "bad" each with corresponding values for $m$ in the fitness function - together with three possible offspring sizes - "small", "medium", and "large". To compare our results to those of McGinley et al., we performed a 
1 simulation experiment that allows only two possible water levels--"good" and "bad"--that

2 occur with probabilities $p_{\mathrm{G}}$ and $p_{\mathrm{B}}=1-p_{\mathrm{G}}$ respectively. McGinley et al. computed the

3 geometric fitness of strategies for various values of $p_{\mathrm{G}}$ and various possible differences in

4 quality between good and bad environments ( $k$ in their notation) and found that a variable-size

5 strategy was favored only for sufficiently large $k$ (large difference in environment quality) and

6 sufficiently large $p_{\mathrm{G}}$. In our model, we choose the "good" and "bad" environments based on

7 results from the experiments. We chose the "good" environment to be $w=16.9 \mathrm{~cm}$, yielding

$8 m=0.60$ and $\alpha=0.22$ (equivalent to $k=1$ in the notation of McGinley et al.). For "bad"

9 environments we looked at $w=21.8,31.5,41.2,50.9,60.6$, and $70.4 \mathrm{~cm}$ (equivalent to $k=2,4$,

$106,8,10,12$ in the notation of McGinley et al.) with corresponding values for $m_{\mathrm{w}}$ and $\alpha_{\mathrm{w}}$ from

11 equations 8 and 9.

13 When we allow environmental quality to be either "good" or "bad" in this way, the single-size 14 strategy wins more often (Fig. 5), which is consistent with what McGinley et al. found. This 15 suggests that the success of the lognormal strategy derives from the fact that real-world 16 environmental conditions are continuously distributed such that there is a nonzero probability 17 of a year with arbitrarily poor conditions. When environmental conditions are distributed as a 18 continuous random variate with nonzero probability for all possible environmental conditions, 19 then for any given tuber size, there must eventually occur a year in which water levels are too 20 deep for the organism to survive. Hence a genet adopting a single-size strategy would 21 eventually die due to a rare but unfavorable year in which water levels were excessive. By 22 comparison, for a genet adopting a lognormal strategy, there is a greater probability of a few 23 large tubers being produced that can survive arbitrarily deep water levels. 
1 As in McGinley et al (1987), the single-size strategy wins when $k$ is small but the variable-

2 size strategies win more often as $k$ increases (Fig. 5). The main difference between our results

3 and those of McGinley et al. is that the variable-size strategy does better when $p_{\mathrm{G}}$ is larger in

4 their model but when $p_{\mathrm{G}}$ is smaller in our model. When $k$ is small (such that the difference

5 between "bad" and "good" years is small) or when $p_{\mathrm{G}}$ is large (such that "bad" years are rare),

6 the lognormal strategy does worse than the single-size strategy because it needlessly invests

7 resources in large tubers. Such a strategy would work well when $k$ is large or when $p_{\mathrm{G}}$ is

8 small, but when "bad" years are rare, it is more efficient to invest resources in producing large

9 numbers of small tubers that are guaranteed to succeed. The lognormal distribution is more

10 successful than the single size distribution for larger $k$ and smaller $p_{\mathrm{G}}$ because the water levels

11 are too deep for any but the largest tubers to survive.

Simulation experiment \#4: fitness in an unchanging environment. The purpose of this experiment was to examine the performance of the lognormal strategy in an environment where the average water level does not change from year to year. For a fixed environmental quality, an optimal tuber size $\mathrm{s}^{*}$ can be obtained from equation 2. At the average water level of $25.5 \mathrm{~cm}$ observed from field conditions (equation 7) equation 2,8 and 9 yield $\mathrm{s}^{*}=0.332 \mathrm{~g}$. For simulations where the water level $\mathrm{w}$ is fixed at $25 \mathrm{~cm}$, we plotted the fitness of the lognormal strategy with mean tuber size $\square=\mathrm{s}^{*}=0.332 \mathrm{~g}$, for various values of $\sigma$. For $\sigma=0$ the lognormal distribution reduces to a single-size strategy. We repeat this with mean tuber size

$21 \square=0.565 \mathrm{~g}$ as computed from equation 10. The results for both comparisons are given in Fig. 22 A3 (Supporting Information). As expected, the geometric mean fitness $\mathrm{K}$ decreases with increasing $\sigma$ in both cases, since there is no advantage to a variable-size strategy when environmental conditions are constant. The global maximum fitness is 885.6 and is achieved by the single-size strategy with mean tuber size $\square=0.565 \mathrm{~g}$. We note that this is higher than the optimal tuber size of $\mathrm{s}^{*}=0.332 \mathrm{~g}$ predicted from equation 2 . 


\section{Discussion}

4 A model by Smith and Fretwell (1974) predicted that, in an unchanging environment, the optimal strategy is to produce propagules of a single size. Subsequently, modeling analyses

6 (some using empirical data to parameterize model) have investigated whether strategies such 7 as dormancy and the production of variably sized offspring might represent adaptive responses to temporally varying environments (see Introduction for references). Most modeling analyses of offspring size distribution strategies have found that producing variably size offspring confers higher fitness than a single-size strategy in variable environments under

11 many scenarios. Here, we presented and analyzed experimental data from the clonal 12 macrophyte species Scirpus maritimus showing that the distribution of offspring tuber sizes is 13 highly variable and best described by a lognormal distribution. The experiments also showed 14 that the distribution was lognormal even under fixed experimental conditions, suggesting that 15 production of variably sized offspring is a developmentally programmed syndrome. We then 16 used a mathematical model parameterized with empirical data to illustrate how production of 17 variably sized offspring tubers can lead to higher fitness than a single-size strategy, by acting as a buffer against temporal environmental variability. This was illustrated using four simulation experiments. The first experiment showed that, at the empirical parameter values controlling the investment strategy in offspring sizes, the fitness under the lognormal strategy

21 was higher than the fitness under the other two strategies. The second experiment showed 22 how the empirical parameter values lie near the global maximum in the fitness landscape of a 23 genet adopting a lognormal strategy. The third experiment showed that a single-size strategy 24 can outperform the lognormal strategy when environmental quality is sampled from a small, 25 restricted set of possible states, but not when it is sampled from a continuous distribution of 
1 possible states where there is a nonzero probability of arbitrarily poor environmental states.

2 The fourth experiment confirmed that the single-size strategy does best when environmental 3 quality does not change over time.

5 The success of the lognormal strategy over the other two strategies relies upon classic within6 generation bet hedging (Hopper 2003) and the reasons for its success can be understood $a$ 7 posteriori by considering the model equations. Under the single-size strategy, for a given tuber size $s$, there is a $100 \%$ probability that a water level sufficiently deep to preclude any

9 biomass production eventually occurs, i.e. $s<m_{\mathrm{w}}$ in equation 1 . This is because $f(w)>0$ for $10 \quad$ all $w$ in equation 7 and so any given water level $w$ will eventually occur if enough time passes. 11 Hence, for any given offspring tuber size under a single-size strategy, there will eventually 12 occur a water level sufficiently deep to kill the genet. We speculate that dormancy will 13 mitigate this effect but may not remove it altogether: a string of sufficiently bad years also 14 occurs eventually with $100 \%$ probability, and hence a dormant tuber may not be able to 15 emerge in a favorable year before becoming unviable or being removed through other 16 processes. Hence, in this model system, single-size strategies are prone to extinction. By comparison, for a large genet adopting a lognormal strategy, for any given water level $w$, there is a nonzero probability of having offspring tubers large enough to survive a year of arbitrarily poor quality $\left(s>m_{\mathrm{w}}\right)$, since for all values of $w$ and $s$. This effect does not occur when environmental states are sampled from a small, discrete set (see Simulation Set \#3 21 Results). The observation of a lognormal distribution in tuber sizes, as opposed to some other common 24 statistical distribution with nonzero variance, is not unexpected. Lognormal distributions are common and have been documented in such diverse phenomena as the critical dose of a drug, 
1 incubation periods of diseases, time to recovery from illness, time to failure of electronic and

2 mechanical devices, times to marriage, divorce, and death, cloud sizes, the abundance of

3 biological species, velocities in air, time required to solve a research problem, and the lengths

4 of telephone calls (Crow \& Shimizu 1988, Haley and Inchausti 2002). It has been shown

5 (Crow and Shimizu 1988, Limpert et. al. 2001) that lognormal distributions should be

6 expected in phenomena that are the result of a multiplicative product of various factors,

7 because the distribution of such outcomes is governed by the multiplicative central-limit

8 theorem. Tuber production in S. maritimus appears to be a multiplicative process rather than

9 an additive one, as the size of new tubers produced along a rhizome in a given season is a

10 function of tubers previously produced along that rhizome in that season as well as previous

11 seasons. Furthermore, if the change in a variate obeys the law of proportionate effect (the

12 change at any step in the process is a random proportion of the previous value), then the

13 variate will tend to be lognormally distributed. This is also true of $S$. maritimus tuber

14 production. These factors may explain why S. maritimus tuber size is lognormally distributed

15 rather than according to some other continuous probability distribution with nonzero

16 variance. If a lognormal distribution may be the product of a complex set of processes not

17 directly relating to fitness, a lognormal distribution per se cannot be called adaptive.

18 However, it is adaptive in the sense that the species may exploit the fact that a lognormal

19 distribution entails production of variably sized offspring. Moreover, it may be possible for a

20 species to evolve with respect to some of the processes that determine the variance of the

21 lognormal distribution. Hence, the variance of the distribution may evolve until the optimal

22 value $\sigma \approx 0.8$ is reached.

24 As with any theoretical model, there are several limitations to the present model. For instance, the model does not take into account the possibility that tubers may remain dormant 
1 for several years before sprouting. Dormancy and density dependence were not incorporated

2 due to lack of empirical data on those aspects of S. maritimus growth. As noted above, 3 dormancy should mitigate the effects of temporal variability in the environment since a

4 portion of tubers can remain dormant during bad years. This should change the quantitative 5 results. However, we speculate that this would not change the relative fitness of the 6 strategies, since dormancy would benefit all strategies to a similar extent. (Just as the 7 probability of an arbitrarily bad year eventually occurring is $100 \%$, so is the probability of a string of arbitrarily bad years, and therefore a single-size strategy remains more prone to

9 failure than a variable-size strategy.) Nonetheless, incorporating dormancy into this model 10 system should be an important priority for future work. Other model limitations include the 11 lack of density dependence in growth dynamics (again, this should change quantitative but not qualitative outcomes) and the use of a continuous model instead of an individual-based model. An individual-based (agent-based) model would allow us to account for discrete effects and 14 thus may be a topic of future research.

16 Though we have focused primarily on temporal heterogeneity in water level fluctuations as the source of diversifying selection, S. maritimus tubers are obviously subject to many other different selective pressures that could interact to produce a diversity of tuber sizes in the

19 population. For example, it is well known, that $S$. maritimus tubers are predated upon by 20 diving ducks (Desnouhes et al. 2007). These predators may selectively remove larger tubers 21 from the population, influencing the size distribution. Our results obviously do not preclude the possibility that the observed tuber size distribution in S. maritimus could arise from other

23 explanations and a multitude of mechanisms. However, it is striking that the lognormal 24 distribution appears both in the field data, which is likely to be the result of many years of 25 selective pressure on more than one genet, and also clearly within a genet in one season (from 
1 the experiments), strongly suggesting a genetic component to this variation. Hangebroek and

2 Santamaria (2004) have studied Potomogeton pectinatus, another aquatic macrophyte which

3 reproduce via tubers and displays considerable size variation (though they do not explicitly

4 discuss the size distribution and nature of variability). They suggest that the size variation is

5 maintained through the interaction of maternal and genetic effects. They find that maternal

6 effects play a major role in determining the size of tubers produced and note that these effects can result in an amplification of within-clone variation in tubers size across generations. As explained previously, this amplification effect is a well-known mechanism for producing lognormally distributed offspring. Hangebroek and Santamaria claim that this effect alone is sufficient to maintain variation in the population and reduce selection pressure towards an optimal tuber size, however, they do not discuss the link between these effects and the offspring size distribution.

In summary, this study supports the hypothesis that production of variably sized offspring in species like S. maritimus can increase fitness by acting as a buffer or bet-hedge against unpredictable temporal variation in an organism's environmental quality, and hence may be adaptive. This work illustrates the benefits of integrating mathematical modeling and empirical approaches in the same investigative framework.

\section{Acknowledgements}

22 M.A. and C.T.B. are supported by the Natural Sciences and Engineering Research Council of

23 Canada (NSERC). M.A. is also supported by a Canada Research Chair. A.C. thanks Josef F.

24 Stuefer for his help in implementing the experiments at the University of Utrecht during

25 which time she was funded by a postdoctoral fellowship from the European Science 
1 Foundation. 


\section{References}

2

3 Charpentier, A., Mesléard, F. \& Thompson, J. D. (1998) The effects of rhizome severing on

the clonal growth and clonal architecture of S. maritimus. Oikos, 83, 107-116.

Charpentier A. \& Stuefer J.F. (1999) Functional specialization of ramets in S. maritimus : Splitting the tasks of sexual reproduction, vegetative growth and resource storage. Plant Ecology, 141, 129-136.

Clevering, O.A. (1995) Life-history characteristics of S. maritimus lacustris and S. maritimus , with special reference to the restoration of these species in former tidal areas. $\mathrm{PhD}$ thesis, Wageningen, The Netherlands

Clevering, O.A., Van Vierssen, W. \& Blom, C.W.P.M. (1995) Growth, photosynthesis and carbohydrate utilization in submerged S. maritimus L. during spring growth. New Phytologist, 130, 105-116.

Crow, E.L. \& Shimizu, K. (1988) Lognormal Distributions: theory and applications. Dekker, New York.

Denouhes, L, Gouraud, C., Lepley, M., Pichaud, M., Guilleman, M. \& Mesléard, F. (2007) Greylag geese (Anser aser)-Scirpus maritium relationships in a newly colonized wintering area in the Camargue (France). Ornis Fennica, 84,12-20.

Einum, S. \& Fleming, I.A. (2000) Highly fecund mothers sacrifice offspring survival to maximaze fitness. Nature, $\mathbf{4 0 5}, 565-567$

Einum, S. \& Fleming, I.A. (2004) Environmental unpredictability and offspring size: conservative versus diversified bet-hedging. Evolutionary Ecology Research, 6, 443455. 
1 Evans M.E.K., Ferrière R., Kane M.J. \& Venable D.L. (2007) Bet hedging via seed banking in desert evening primroses (Oenothera, Onagraceae): Demographic Evidence from Natural Populations. American Naturalist, 169(2), 184-194.

Forbes L.S. (1999) Within-clutch variation in propagule size: the double-fault model. Oikos, 85, 146-150.

Fox C.W., Thakar M.S. \& Mousseau T.A. (1997) Egg size plasticity in a seed beetle: an adaptive maternal effect. American Naturalist, 149,149-163.

Geritz S.A.H. (1995) Evolutionary stable seed polymorphism and small-scale spatial variation in seedling density. American Naturalist, 146, 685-707.

Geritz, S.A.H., E. van der Meijden \& J.A.J. Metz. (1999) Evolutionary dynamics of seed size and seedling competitive ability. Theoretical Population Biology, 55, 324-343.

Gómez J.M. (2004) Bigger is not always better: conflicting selective pressures on seed size in Quercus ilex. Evolution, 58(1), 71-80.

Grace J.B. (1989) Effect of water depth on Typha latifolia and Typha domingensis. American Journal of Botany, 76, 762-768.

Hairston N.G., Munns W.R. (1984) The timing of copepod diapause as an evolutionarily stable strategy. American Naturalist, 123(6), 733-751.

Halley, J. \& Inchausti, P. (2002) Lognormality in ecological time series. Oikos, 99(3), 518530.

Hangelbroek, H.H. \& Santamaria, L. (2004) Regulation of propagule size in the aquatic pseudo-annual Potamogeton pectinatus: are genetic and maternal non-genetic effects additive? Evolutionary Ecology Research, 6, 147-161

Hendry A.P., Day T. \& Cooper A.B. (2001) Optimal size and number of propagules: Allowance for discrete stages and effects of maternal size on reproductive output and offspring fitness American Naturalist, 157, 387-407. 
1 Hopper K.R., Rosenheim J.A., Prout T. \& Oppenheim S.J. (2003) Within-generation bethedging: a seductive explanation? Oikos, 101, 219-222.

3 Kalisz S.\& McPeek, M.A. (1993) Extinction dynamics, population growth and seed banks. Oecologia, 95, 314-320.

Kantrud, H. A. (1996) The alkali (S. maritimus L.) and saltmarsh (S. robustus Pursh)

Kaplan R.H. \& Cooper W.S. (1984) The evolution of developmental plasticity in reproductive characteristics: an application of the "adaptive coin-flipping" principle. American Naturalist, 123, 393-410.

Kisdi E. (2007) No direct selection to increase offspring number of bet-hedging strategies in large populations: Simons' model revisited. Journal of Evolutionary Biology, 20, 2072-2074.

Kudo S. (2001) Intraclutch egg-size variation in acanthosomatid bugs: adaptive allocation of maternal investment? Oikos, 92, 208-214.

Lalonde R.G. (1991) Optimal offspring provisioning when resources are nor predictable. American Naturalist, 138, 680-686.

Lieffers, V.J. \& Shay, J.M. (1982) Seasonal growth and standing crop of S. maritimus var. paludosus in Saskatchewan. Canadian Journal of Botany, 60, 117-125.

Limpert, E., Stahel, W.A. \& Abbt, M. (2001) Lognormal distributions across the sciences: keys and clues. Bioscience, 51, 341-352.

Marshall, D.J., Cook, C.N. \& Emlet, R.B. (2006) Offspring size effects mediate competitive interactions in a colonial marine invertebrate. Ecology, 87(1), 214-225. 
1 Marshall, D.J., Bonduriansky, R., \& Bussière, L.F. (2008) Offspring size variation within broods as a bet-hedging strategy in unpredictable environments. Ecology, 89(9), 2506-2517.

McGinley M.A., Temme D.H. and Geber M.A. (1987) Parental investment in offspring in variable environments: theoretical and empirical considerations. American Naturalist, 130, 370-398.

Moles A.T., Warton D.I., Westoby M. (2003) Do small-seeded species have higher survival through seed predation than large-seeded species? Ecology, 84(12), 3148-3161.

Moles A.T. and Westoby M. (2006) Seed size and plant strategy across the whole life cycle. Oikos, 113, 91-105.

Olofsson H., Ripa J., Jonzén N (2009) Bet-hedging as an evolutionary game: the trade-off between egg size and number. Proceedings of the Royal Society of London B, 276, 2963-2969.

Parker G.P. and Begon M. (1986) Optimal egg size and clutch size: effects of environment and maternal phenotype. American Naturalist, 128, 573-592

Peck, S.L. (2004) Simulation as experiment: a philosophical reassessment for biological modeling. Trends in Ecology and Evolution, 19(10), 530-534.

Philippi T. and Seger J. (1989) Hedging one's evolutionary bets, revisited. Trends in Ecology and Evolution, 4(2), 41-44.

Sakai S. (1995) A model for seed size variation among plants. Evolutionary Ecology, 9, 495507.

Sasaki A. and Ellner S. (1995) The evolutionarily stable phenotype distribution in a random environment. Evolution, 49, 337-350.

Schultz D. (1991) Parental investment in temporally varying environments. Evolutionary Ecology, 5, 415-427. 
1 Simons A.M. and Johnston M.O. (1997) Developmental instability as a bet-hedging strategy. Oikos, 80, 401-406

Simons A.M. and Johnston M.O. (2000) Variation in seed traits of Lobelia inflata (Campanulaceae): sources and fitness consequences. American Journal of Botany, 87, 124-132.

Simons A.M. and Johnston M.O. (2006) Environmental and genetic sources of diversification in the timing of seed germination: implications for the evolution of bet hedging. Evolution, 60(11), 2280-2292.

Simons A.M. (2007) Selection for increased allocation to offspring number under environmental unpredictability. Journal of Evolutionary Biology, 20, 813-817.

Smith C.C. and Fretwell S.D. (1974) The optimal balance between size and number of offspring. American Naturalist, 108, 499-506.

Spence D.H.N. (1982) The zonation of plants in freshwater lakes. Advances in Ecological Research, 12, 37-125.

Stearns S.C. (1992) The evolution of life histories. Oxford University Press, Oxford.

Torres-Vila L.M. and Rodriguez-Molina M.C. (2002) Egg size variation and its relationship with larval performance in the Lepidoptera: the case of the European grapevine moth Lobesia botrana. Oikos, 99, 272-283.

Vaughton G. and Ramsey M. (1998) Sources and consequences of seed mass variation in Banksia marginata (Peoteaceae). Journal of Ecology, 86, 563-573.

Venable D.L. (1992) Size-number trade-offs and the variations of seed size with plant resource status. American Naturalist, 140, 287-304.

Venable D.L. and Brown J.S. (1988) The selective interactions of dispersal, dormancy and seed size as adaptations for reducing risk in variable environments. American Naturalist, 1, 360-384. 
1 Venable D.L. (2007) Bet hedging in a guild of desert annuals. Ecology, 88(5), 1086-1090.

2 Vinuela J. (1997) Adaptation vs. constraint: intracluch egg-mass variation in birds. Journal of 3 Animal Ecology, 66, 781-792

4 Westoby, M., M.R. Leishman and J.M. Lord. (1996) Comparative ecology of seed size and 5 seed dispersal. Philosophical Transactions of the Royal Society B, 351, 1309-1318.

6 Wolfe L.M. (1995) The genetics and ecology of seed size variation in a biennial plant, 7 Hydrophyllum appendiculatum (Hydrophyllaceae). Oecologia, 101, 343-352. 8 
1 Table 1. Fit of test distributions to individual ramet data in shallow, medium, and deep water

2 levels*

\begin{tabular}{|c|c|c|c|c|c|}
\hline & & & $\begin{array}{r}\text { Distril } \\
\text { Statisti }\end{array}$ & $\begin{array}{l}\text { Ition Fitted } \\
\text { l Test Used }\end{array}$ & \\
\hline & & $\begin{array}{l}\text { Lognormal } \\
\text { KSL }\end{array}$ & $\begin{array}{l}\text { Lognormal } \\
\text { Shapiro-Wilks }\end{array}$ & $\begin{array}{c}\text { Normal } \\
\text { Shapiro-Wilks }\end{array}$ & $\begin{array}{c}\text { Gamma } \\
\text { Cramer v. Mises }\end{array}$ \\
\hline $\begin{array}{l}\text { Shallow } \\
(15 \mathrm{~cm})\end{array}$ & $N=29$ & $\begin{array}{l}\text { REJECT } \\
D=0.179 \\
p>D=0.03 \\
\mu=-0.716 \\
\sigma=0.741\end{array}$ & $\begin{array}{l}\text { REJECT } \\
W=0.908 \\
p<W=0.03 \\
\mu=-0.716 \\
\sigma=0.741\end{array}$ & $\begin{array}{l}\text { REJECT } \\
W=0.919 \\
p<W=0.02 \\
\mu=0.626 \\
\sigma=0.418\end{array}$ & $\begin{array}{l}\text { REJECT } \\
\text { No Convergence }\end{array}$ \\
\hline & $N=25$ & $\begin{array}{l}\text { ACCEPT } \\
D=0.128 \\
p>D=0.15 \\
\mu=-0.776 \\
\sigma=0.955 \\
\text { ACCEPT } \\
D=0.104 \\
p>D=0.15 \\
\mu=-0.708 \\
\sigma=0.777\end{array}$ & $\begin{array}{l}\text { ACCEPT } \\
W=0.944 \\
p<W=0.182 \\
\mu=-0.776 \\
\sigma=0.955 \\
\text { ACCEPT } \\
W=0.942 \\
p<W=0.221 \\
\mu=-0.708 \\
\sigma=0.795\end{array}$ & $\begin{array}{l}\text { REJECT } \\
W=0.877 \\
p<W=0.006 \\
\mu=0.679 \\
\sigma=0.574 \\
\text { BORDERLINE } \\
\text { ACCEPT } \\
W=\mathbf{0 . 9 1 3} \\
\boldsymbol{p}<W=\mathbf{0 . 0 5 3} \\
\boldsymbol{\mu}=\mathbf{0 . 6 4 5} \\
\sigma=\mathbf{0 . 4 4 7}\end{array}$ & $\begin{array}{l}\text { REJECT } \\
\text { No Convergence }\end{array}$ \\
\hline & $\begin{array}{l}\text { All } \\
N=398\end{array}$ & $\begin{array}{l}\text { REJECT } \\
D=0.05 \\
p>D=0.012 \\
\mu=-0.788 \\
\sigma=0.837\end{array}$ & $\begin{array}{l}\text { REJECT } \\
\mathrm{W}=0.988 \\
p<W=0.002 \\
\mu=-0.788 \\
\sigma=0.837\end{array}$ & REJECT & $\begin{array}{l}\text { REJECT } \\
\text { No Convergence }\end{array}$ \\
\hline $\begin{array}{l}\text { Medium } \\
(45 \mathrm{~cm})\end{array}$ & $N=14$ & $\begin{array}{l}\text { ACCEPT } \\
D=0.200 \\
p>D=0.128 \\
\mu=-1.17 \\
\sigma=0.906\end{array}$ & $\begin{array}{l}\text { АCСЕРТ } \\
W=0.912 \\
p<W=0.167 \\
\mu=-1.17 \\
\sigma=0.940\end{array}$ & $\begin{array}{l}\text { BORDERLINE } \\
\text { ACCEPT } \\
W=0.897 \\
p<W=0.101 \\
\mu=0.624 \\
\sigma=0.495\end{array}$ & $\begin{array}{l}\text { REJECT } \\
\text { No Convergence }\end{array}$ \\
\hline & $N=16$ & $\begin{array}{l}\text { ACCEPT } \\
D=0.143 \\
p>D=0.15 \\
\mu=-0.769 \\
\sigma=0.805\end{array}$ & $\begin{array}{l}\text { ACCEPT } \\
W=0.949 \\
p<W=0.467 \\
\mu=-0.769 \\
\sigma=0.831\end{array}$ & $\begin{array}{l}\text { REJECT } \\
W=0.867 \\
p<W=0.03 \\
\mu=0.435 \\
\sigma=0.312\end{array}$ & $\begin{array}{l}\text { REJECT } \\
\text { No Convergence }\end{array}$ \\
\hline & $N=13$ & $\begin{array}{l}\text { ACCEPT } \\
D=0.102 \\
p>D=0.15 \\
\mu=-1.00\end{array}$ & $\begin{array}{l}\text { ACCEPT } \\
W=0.914 \\
p<W=0.206 \\
\mu=-0.126\end{array}$ & $\begin{array}{l}\text { REJECT } \\
W=0.853 \\
p<W=0.03 \\
\mu=0.428\end{array}$ & $\begin{array}{l}\text { REJECT } \\
\text { No Convergence }\end{array}$ \\
\hline & $\begin{array}{l}\text { All } \\
N=210\end{array}$ & $\begin{array}{l}\sigma=0.538 \\
\text { ACCEPT } \\
D=0.05\end{array}$ & $\begin{array}{l}\sigma=0.787 \\
\text { ACCEPT } \\
W=0.989\end{array}$ & $\begin{array}{l}\sigma=0.263 \\
\text { REJECT } \\
W=0.870\end{array}$ & $\begin{array}{l}\text { REJECT } \\
\text { No Convergence }\end{array}$ \\
\hline
\end{tabular}




\begin{tabular}{|c|c|c|c|c|c|}
\hline \multirow{19}{*}{$\begin{array}{l}\text { Deep } \\
(85 \mathrm{~cm})\end{array}$} & \multirow{8}{*}{$N=10$} & $p>D=0.15$ & $p<W=0.1$ & $p<W=0.001$ & \multirow{8}{*}{$\begin{array}{l}\text { REJECT } \\
\text { No Convergence }\end{array}$} \\
\hline & & $\mu=-1.06$ & $\mu=-1.06$ & $\mu=0.459$ & \\
\hline & & $\sigma=0.786$ & $\sigma=0.787$ & $\sigma=0.347$ & \\
\hline & & ACCEPT & ACCEPT & BORDERLINE & \\
\hline & & $D=0.73$ & $W=0.924$ & ACCEPT & \\
\hline & & $p>D=0.15$ & $p<W=0.392$ & $W=0.85$ & \\
\hline & & $\mu=-1.39$ & $\mu=-1.39$ & $p<W=0.057$ & \\
\hline & & $\sigma=0.973$ & $\sigma=1.02$ & $\mu=0.387$ & \\
\hline & \multirow{4}{*}{$N=10$} & & & $\sigma=0.366$ & \multirow{4}{*}{$\begin{array}{l}\text { REJECT } \\
\text { No Convergence }\end{array}$} \\
\hline & & ACCEPT & ACCEPT & ACCEPT & \\
\hline & & $D=0.128$ & $W=0.936$ & $W=0.956$ & \\
\hline & & $p>D=0.15$ & $p<W=0.51$ & $p<W=0.7428$ & \\
\hline & \multirow{7}{*}{$\begin{array}{l}\text { All } \\
N=94\end{array}$} & $\mu=-1.44$ & $\mu=-1.44$ & $\mu=0.302$ & \multirow{7}{*}{$\begin{array}{l}\text { REJECT } \\
\text { No Convergence }\end{array}$} \\
\hline & & $\sigma=0.775$ & $\sigma=0.817$ & $\sigma=0.194$ & \\
\hline & & ACCEPT & ACCEPT & REJECT & \\
\hline & & $D=0.07$ & $W=0.984$ & $W=0.720$ & \\
\hline & & $p>D=0.15$ & $p<W=0.327$ & $p<W=0.001$ & \\
\hline & & $\mu=-1.58$ & $\mu=-1.58$ & $\mu=0.295$ & \\
\hline & & $\sigma=0.824$ & $\sigma=0.828$ & $\sigma=0.296$ & \\
\hline
\end{tabular}

1 * Lognormal fitting used two different methods in order to be able to compare p-values

2 directly with the method commonly used for fitting the normal distribution. In most cases

3 (marked in bold type), the lognormal fit the data tested best. In the few cases where the data

4 was fit by the normal distribution, the lognormal fit was better. None of the data were fit by

5 the gamma distribution. Note that the null hypothesis tested in each case is different,

6 however for ease of presentation ACCEPT/REJECT refers to the null hypothesis that the

7 data belong to the distribution labelled in the respective column. 
1 Table 2. Parameter values used in simulations for simulation experiments 1 through 4

\begin{tabular}{|c|c|c|}
\hline Parameter/Function & Definition & Values \\
\hline & Probability distribution & Simulation set \#1: $a=4.646, b=-$ \\
\hline \multirow[t]{7}{*}{ equation 7} & function for fluctuations & 0.080 \\
\hline & in water level $w$ from year & Simulation set \#2: $a=4.646, b=-$ \\
\hline & to year & 0.080 \\
\hline & & Simulation set\#3: n/a (used only two \\
\hline & & possible water levels) \\
\hline & & Simulation set \#4: n/a (used a \\
\hline & & constant water level of $25 \mathrm{~cm}$ ) \\
\hline \multirow[t]{3}{*}{$\sigma$} & Variance $($ of $\log (S)$ for a & Sets \#1, 3: 0.821 \\
\hline & lognormally distributed & Sets \#2, 4: varies on $[0,1.5]$ \\
\hline & tuber size $S)$ & \\
\hline & Minimum viable offspring & $c=0.010, d=-0.124 \mathrm{~g}$ for all sets \\
\hline \multirow[t]{3}{*}{ equation 8} & size as a function of water & \\
\hline & level $w$ & \\
\hline & Shape parameter for & $e=-0.022, f=0.258$ for all sets \\
\hline \multirow[t]{2}{*}{ equation 9} & fitness function as a & \\
\hline & function of water level $w$ & \\
\hline & Mean tuber size produced & Sets \#1, 3, 4: $y_{\mathrm{int}}=0.680 \mathrm{~g}$ \\
\hline & as a function of water & Set \#2: $y_{\text {int }}$ varies on $[0 \mathrm{~g}, 2 \mathrm{~g}]$ \\
\hline equation 10 & level $w$ & \\
\hline \multirow[t]{2}{*}{$S_{\text {half }}$} & Width parameter for & $0.1 \mathrm{~g}$ for all sets \\
\hline & uniform distribution & \\
\hline \multirow[t]{2}{*}{$T$} & Duration of simulation & 350 years per simulation run for all \\
\hline & runs & sets \\
\hline \multirow[t]{2}{*}{$R$} & Number of realizations & Sets \#1, 2, 4: 8,000 \\
\hline & per set & Set \#3: 5,000 \\
\hline
\end{tabular}



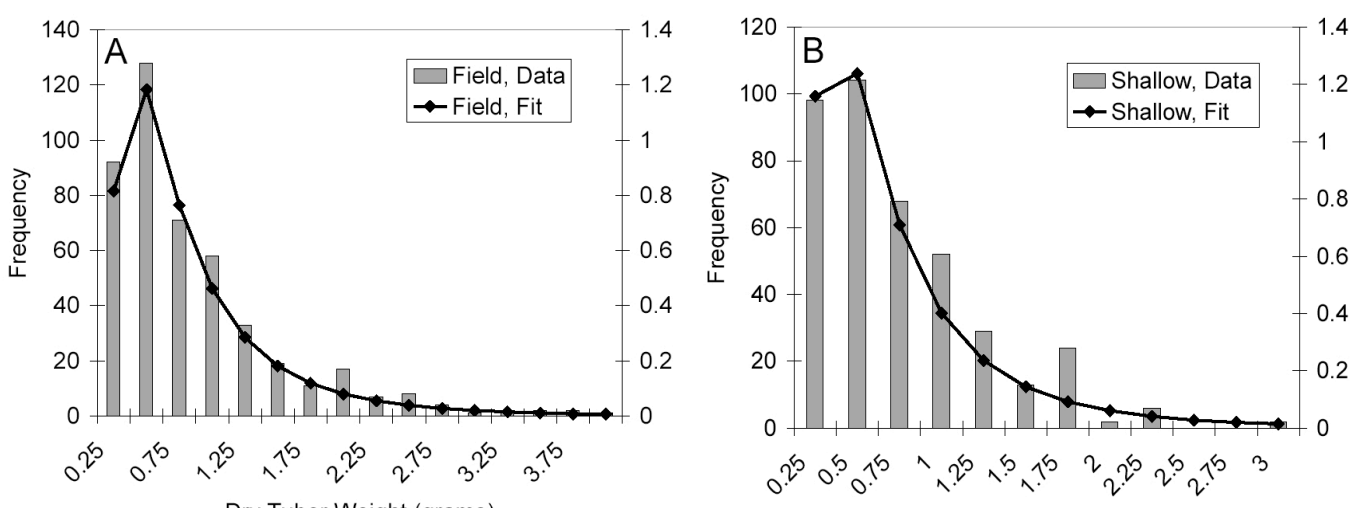

Dry Tuber Weight (grams)
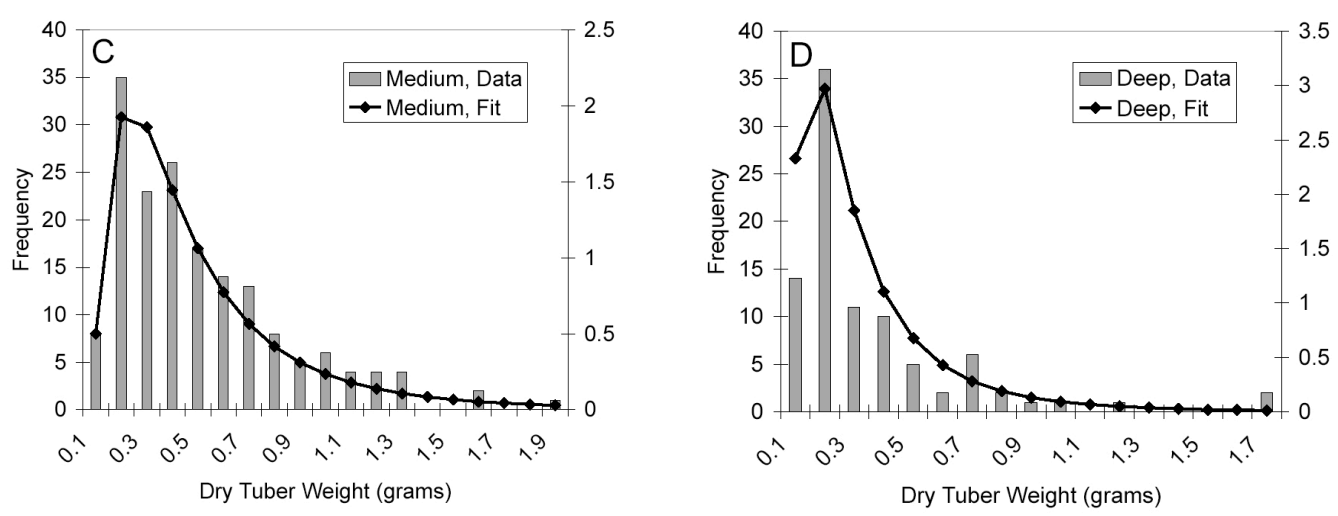

6 Fig. 1. Distribution of tuber sizes (A) collected from the field $(N=455)$ and lognormal fit 7 (Kolmogorov D-test, $\mu=-0.636, \sigma=0.821, \mathrm{D}=0.06, p=0.01$ ); (B) produced in shallow water 8 level $(N=398)$ and lognormal fit (Kolmogorov D-test, $\mu=-0.788, \sigma=0.837, \mathrm{D}=0.05, p=0.01)$;

9 (C) produced in medium water level $(N=210)$ and lognormal fit (Kolmogorov D-test, $10=-1.06, \sigma=0.786, \mathrm{D}=0.05, p=0.15)$; (D) produced in deep water level $(N=94)$ and lognormal 11 fit (Kolmogorov D-test, $\mu=-1.58, \sigma=0.824, \mathrm{D}=0.07, p=0.15$ ). 

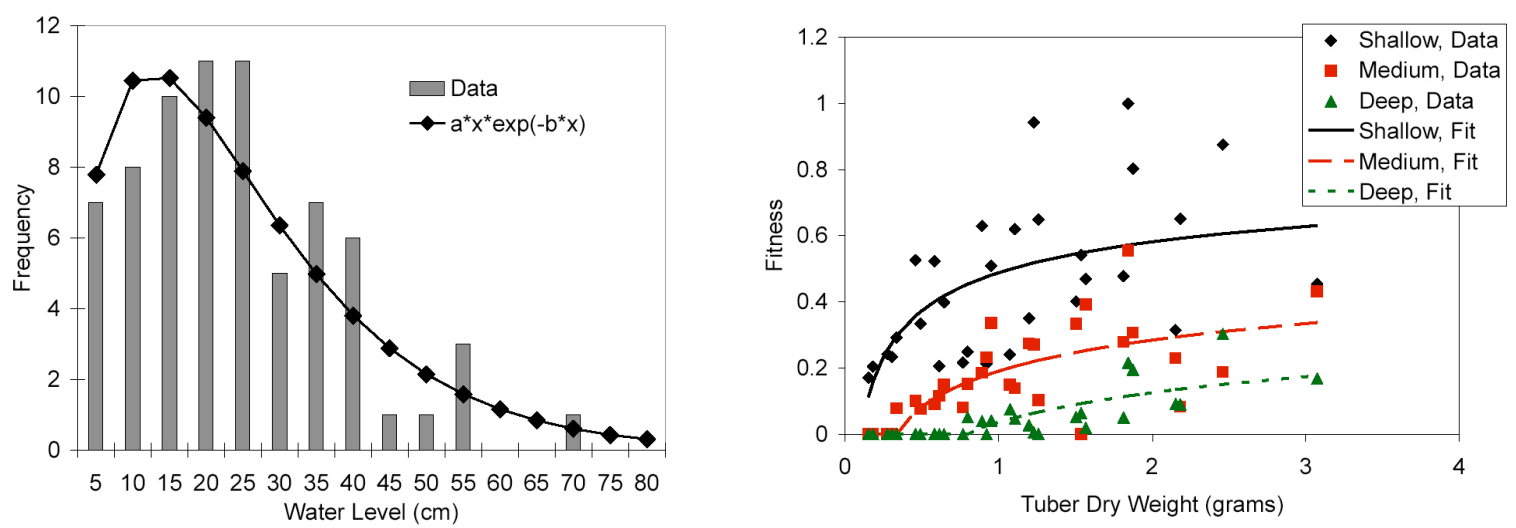

5

6 Fig. 2. (A) Frequency distribution of water level at the end of March over the period 1932-

$72003(n=71)$ and fitted water level distribution function, equation 7 (B) Relationship between

8 the total biomass of produced tubers and the size of planted tubers, with fitted fitness

9 functions, equation 1 , for the three experimental water levels: shallow $(15 \mathrm{~cm})$, medium $(45$ $\mathrm{cm})$ and deep (85 cm). (B).

11

12 

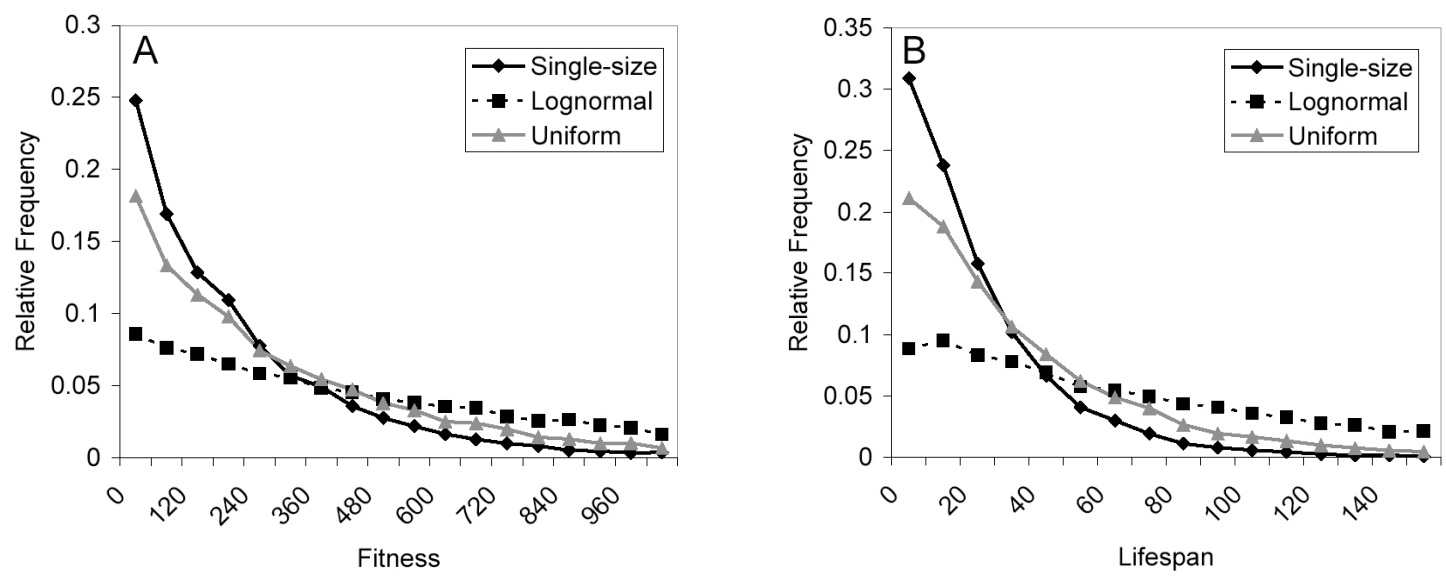

5

6

$7 \quad$ Fig. 3. Frequency distributions of (A) fitness and (B) lifespan for simulation experiment \#1.

8 

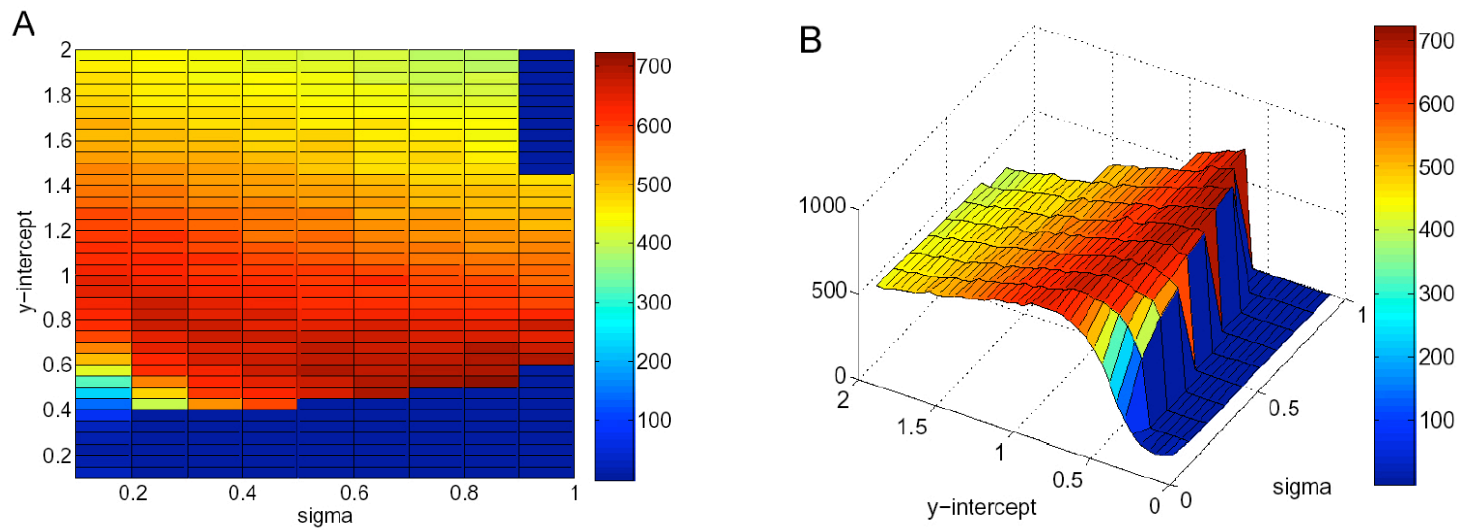

5

6

$7 \quad$ Fig. 4. Fitness landscape for simulation experiment \#2, (C) top view and (D) three-

8 dimensional view. For comparison, note that $\sigma=0.821$ and $y_{\mathrm{int}}=0.680$ at empirical values. 9 


\begin{tabular}{cccccccc}
$\begin{array}{c}\text { Probability } \\
\text { of a Good }\end{array}$ & & \multicolumn{6}{c}{ Difference in environmental quality $(\mathrm{k})$} \\
Year $\left(\mathrm{p}_{\mathrm{G}}\right)$ & $\mathrm{k}=1$ & $\mathrm{k}=2$ & $\mathrm{k}=4$ & $\mathrm{k}=6$ & $\mathrm{k}=8$ & $\mathrm{k}=10$ & $\mathrm{k}=12$ \\
& 0.85 & 0.78 & 0.68 & 0.71 & 1.60 & 80.4 & 1496.9 \\
$\mathrm{p}_{\mathrm{G}}=0$ & 0.85 & 0.78 & 0.69 & 0.73 & 1.51 & 422.0 & 207.1 \\
& 0.85 & 0.78 & 0.70 & 0.72 & 1.40 & 41.8 & 189.8 \\
$\mathrm{p}_{\mathrm{G}}=0.1$ & 0.85 & 0.78 & 0.70 & 0.73 & 1.34 & 120.0 & 63.7 \\
& 0.85 & 0.79 & 0.71 & 0.86 & 1.24 & 27.6 & 90.6 \\
$\mathrm{p}_{\mathrm{G}}=0.2$ & 0.85 & 0.79 & 0.71 & 0.74 & 1.20 & 41.4 & 37.3 \\
& 0.85 & 0.79 & 0.73 & 0.74 & 1.12 & 21.3 & 59.8 \\
$\mathrm{p}_{\mathrm{G}}=0.3$ & 0.85 & 0.79 & 0.73 & 0.75 & 1.09 & 16.7 & 27.8 \\
& 0.85 & 0.80 & 0.75 & 0.75 & 1.03 & 16.3 & 40.8 \\
$\mathrm{p}_{\mathrm{G}}=0.4$ & 0.85 & 0.80 & 0.75 & 0.76 & 1.01 & 7.7 & 20.9 \\
& 0.85 & 0.81 & 0.76 & 0.76 & 0.96 & 12.8 & 29.9 \\
$\mathrm{p}_{\mathrm{G}}=0.5$ & 0.85 & 0.81 & 0.76 & 0.77 & 0.95 & 4.0 & 16.4 \\
& 0.85 & 0.82 & 0.78 & 0.78 & 0.91 & 9.3 & 19.1 \\
$\mathrm{p}_{\mathrm{G}}=0.6$ & 0.85 & 0.82 & 0.78 & 0.78 & 0.90 & 2.3 & 11.6 \\
& 0.85 & 0.83 & 0.79 & 0.79 & 0.87 & 6.3 & 11.6 \\
$\mathrm{p}_{\mathrm{G}}=0.7$ & 0.85 & 0.83 & 0.79 & 0.79 & 0.87 & 1.5 & 7.7 \\
& 0.85 & 0.83 & 0.81 & 0.81 & 0.85 & 3.7 & 5.9 \\
$\mathrm{p}_{\mathrm{G}}=0.8$ & 0.85 & 0.83 & 0.81 & 0.81 & 0.85 & 1.1 & 4.3 \\
& 0.85 & 0.84 & 0.83 & 0.83 & 0.84 & 1.60 & 2.1 \\
$\mathrm{p}_{\mathrm{G}}=0.9$ & 0.85 & 0.84 & 0.83 & 0.83 & 0.84 & 0.92 & 1.8 \\
& 0.85 & 0.85 & 0.85 & 0.85 & 0.85 & 0.85 & 0.85 \\
$\mathrm{p}_{\mathrm{G}}=1.0$ & 0.85 & 0.85 & 0.85 & 0.85 & 0.85 & 0.85 & 0.85
\end{tabular}

5 Fig. 5. Results for simulation experiment \#3. Each cell shows the ratio of lognormal strategy

6 fitness to single-size strategy fitness (top entry in each cell) and lognormal fitness to uniform

$7 \quad$ strategy fitness (bottom entry in each cell), for various values of $p_{\mathrm{G}}$ and $k$. Clear cells (resp.

8 light grey and dark grey cells) denote cases where the single-size strategy (resp. uniform and 9 lognormal) obtained the highest fitness. 


\section{Supporting Information}

2

\section{$3 \quad$ Equations for strategies}

4

5 The three probability density functions for offspring size distributions (single-sized,

6 lognormal, and uniform) are given by

7

8

9

10

11

13

14

15

16

17

18

19

20

21 the equation

$$
\phi_{w}^{\text {single }}(s)=\delta\left(\hat{s}_{w}-s\right)
$$$$
\phi_{w}^{\log }(s)=\frac{1}{s \sqrt{2 \pi} \sigma} \exp \left[-\frac{1}{2 \sigma^{2}}\left(\ln (s)-\ln \left(\hat{s}_{w}\right)-\sigma / 2\right)^{2}\right]
$$

$$
\phi_{w}^{\text {uni }}(s)=\left\{\begin{array}{cc}
1 /\left(2 s_{\text {half }}\right) & s>\hat{s}_{w}-s_{\text {half }} \text { and } s<\hat{s}_{w}+s_{\text {half }} \\
0 & \text { otherwise }
\end{array}\right.
$$

where $\square$ is the mean offspring size in water level $w, \sigma$ is the variance of the lognormal

distribution, and $s_{\text {half }}$ controls the width of the uniform distribution. The integral of these distributions from $s=0$ to $s=\infty$ is 1 .

\section{Derivation of model equation 3}

We first assume that tubers come in discrete sizes $s_{1}=\Delta s, s_{2}=2 \Delta s, s_{3}=3 \Delta s, \ldots$ where $\Delta s$ is a small, fixed value (later we will relax this assumption). The total biomass $b_{\mathrm{t}}$ produced in year $t$ depends upon the sizes of tubers produced in year $t-1$, when the water level was $w(t-1)$, and the total biomass produced by these tubers in year $t$, when the water level is $w(t)$, according to
. 
1 The number of size $s_{\mathrm{k}}$ tubers produced in year $t-1$ is equal to the total number of tubers (of all

2 sizes) produced in year $t-1$ (i.e., total biomass $b_{\mathrm{t}-1}$ produced divided by mean tuber size

3 $\square$ of tubers produced in year $t-1)$, times the fraction that that are size $s_{\mathrm{k}}$. The

4 biomass produced per size $s_{\mathrm{k}}$ tuber in year $t$ is equal to the fitness function corresponding to the water level in year $t$. Hence,

Taking the limit as , one finally obtains

\section{Discrete effects}

13 This model assumes that tuber offspring sizes are distributed according to a continuous

14 function. However, real populations are discrete in nature, consisting of a finite number of 15 distinct individuals. Because of this there exist parameter values for which the assumption of 16 continuity does not hold, and discrete effects become significant. For example, in some years 17 when the water level is unusually deep, it may happen that a tuber fails to produce enough total biomass to make even a single daughter tuber at the sizes required by the mean tuber size Hence we restrict our analysis to parameter values such that these violations are rare. Let $\square$ denote the mean tuber size produced in year $t$. Then, a violation of the continuity assumption 
1 is considered to occur if the total biomass produced by a tuber of size $\square$ in year $t$ is less than

$2 \square$ plus two standard deviations of the lognormal distribution. That is, a violation occurs when 3 tubers with mean required size from the previous year cannot produce even a single tuber, at

4 the mean required size in the present year, in $2.3 \%$ of the cases (since two standard deviations 5 covers $97.7 \%$ of the distribution of produced tuber sizes). It can be shown that this translates 6 into the condition

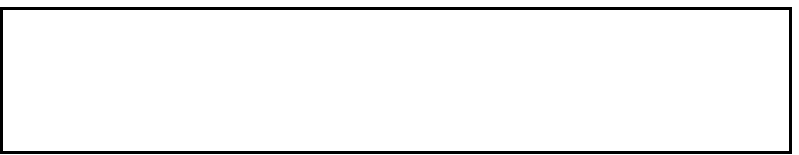

8 Note that, since the lognormal distribution has the largest variance, it is sufficient to apply this

9 criterion to the lognormal strategy only: if one of the other strategies experiences a violation 10 event, then the lognormal strategy must also (although the converse is not true). A 11 particularly deep water level in year $t-1$, or year $t$, or both, tends to produce a violation. We 12 restrict all analysis to parameter values such that years with a violation event constitute no 13 more than $5 \%$ of the years of a given simulation run. 
1 Table A1. Tuber production at the individual level, for the shallow, medium and deep

2 treatment*

3

$4 *$ For each treatment, the dry weight $(\mathrm{g})$ of the planted tuber, the total number of produced 5 tubers $(\mathrm{N})$, the dry weight $(\mathrm{g})$ of the smallest (min) and biggest (max) produced tuber, the median (M), mean and standard deviation (S.D) are presented 


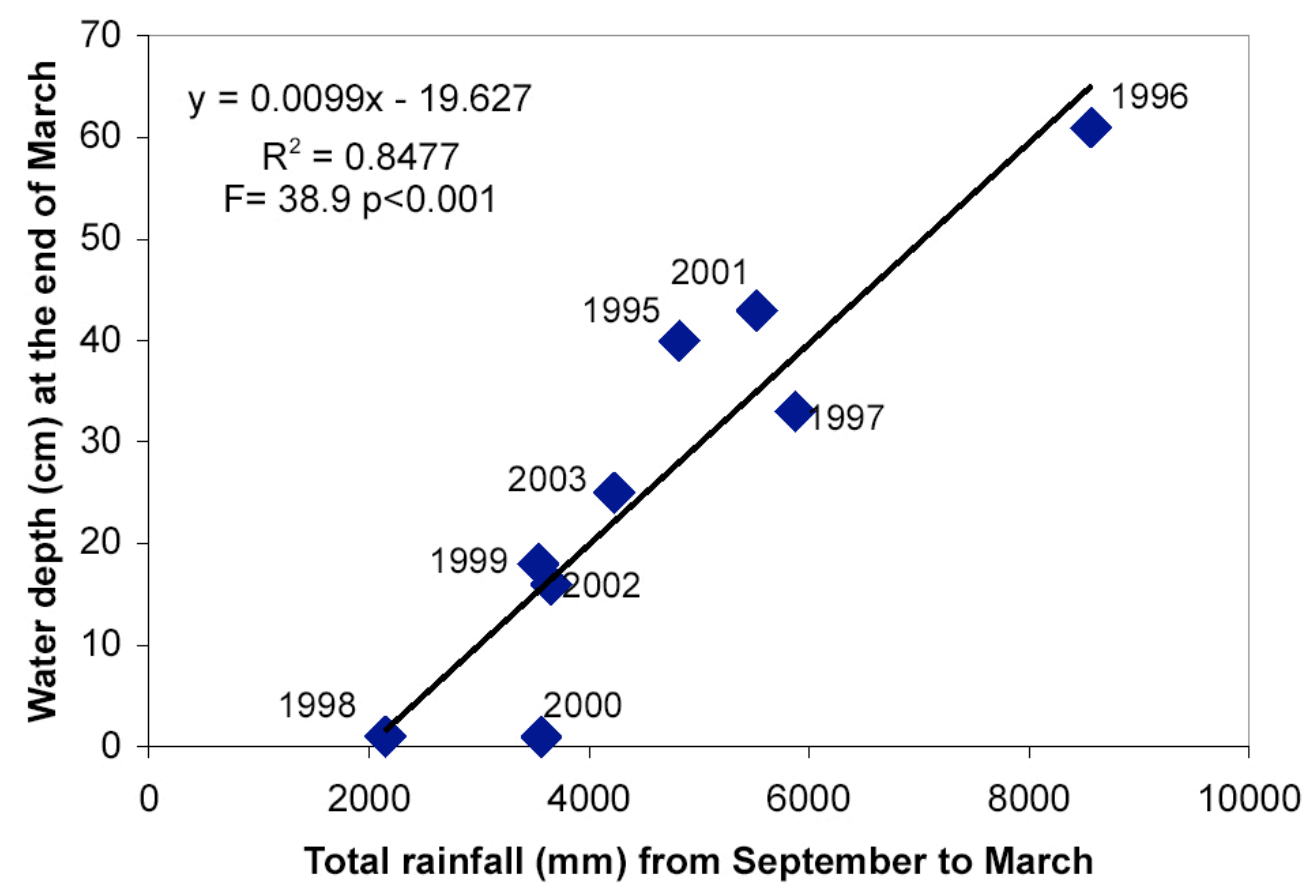

2 Fig. A1. Relationship between rainfall and water level for the period 1995-2003.

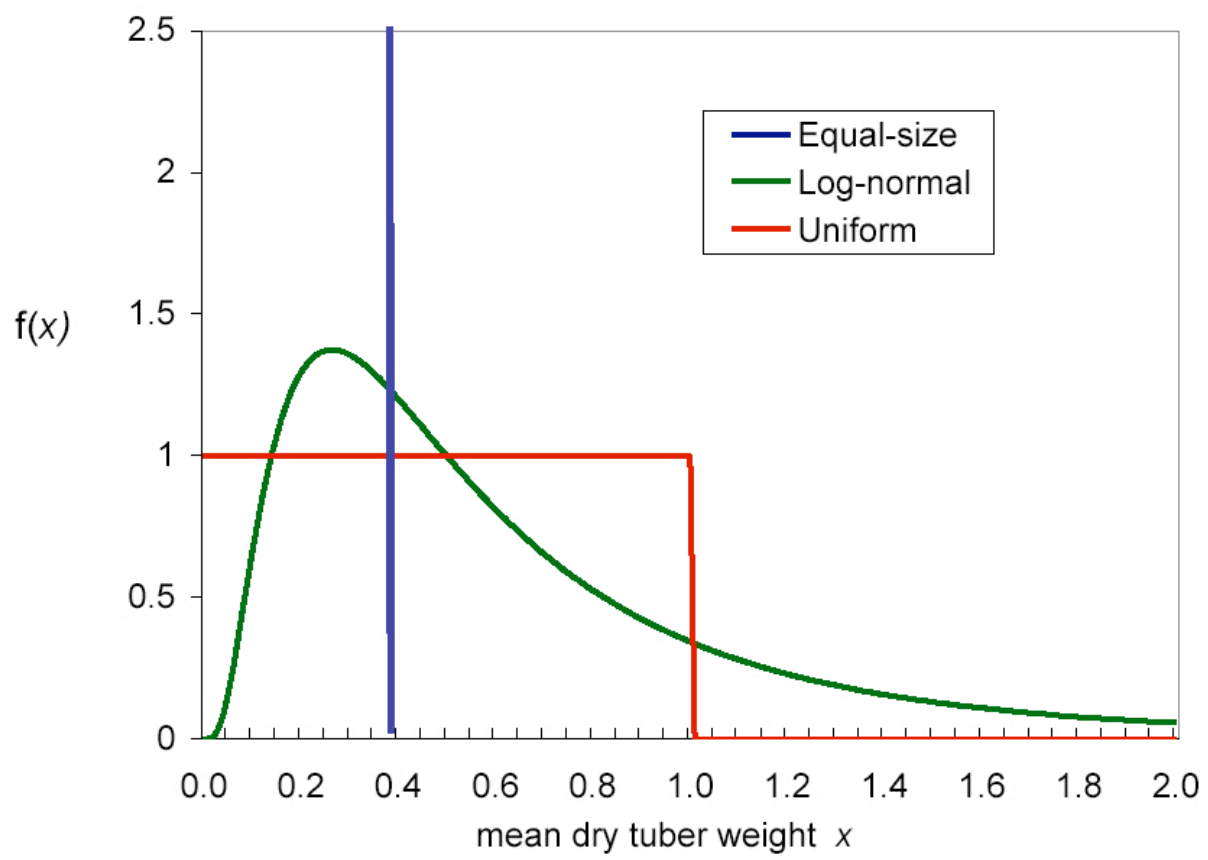

4

5 Fig. A2. Illustration of the three offspring size distribution strategies: lognormal, uniform, and 6 single-size. 


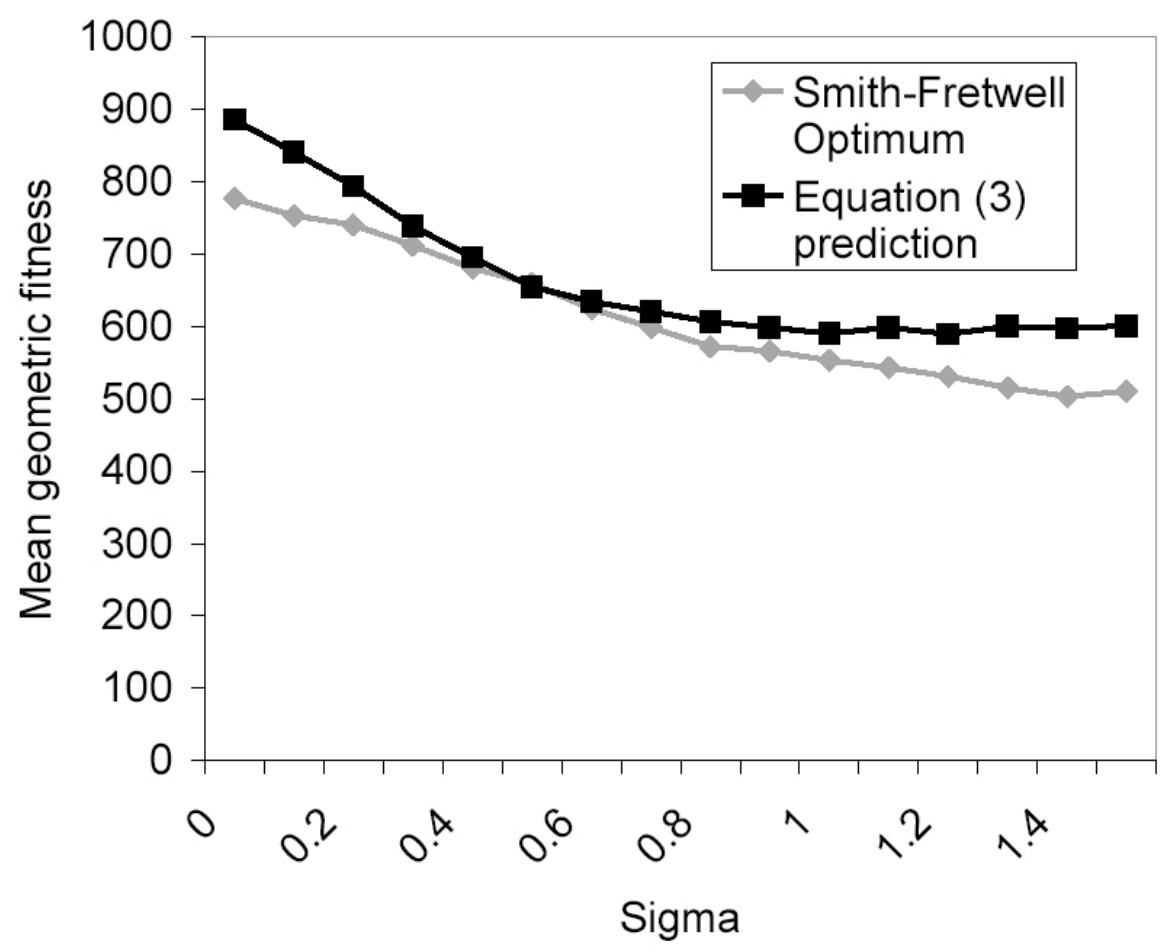

4

$5 \quad$ Fig. A3. Results for simulation experiment \#4: fitness in an unchanging environment for 6 various values of $\sigma$. 